other health issues, there is lack of champion organisation in these countries to work on child injury problem,

Results Effects/Changes Centre for Injury Prevention and Research, Bangladesh (CIPRB) has been evolved in Bangladesh more than a decade back and made a significant changes in the health policy. CIPRB conducted number of child injury intervention researches, notably, PRECISE, SOLID, BASS involving the policy makers from very beginning to create convincing evidences for child injury prevention. With many of its innovation, CIPRB has been engaged in advocacy and communication to make the child injury prevention a priority agenda at national level. As a result, National Child Injury Prevention Strategy formulated, Injury included in Health Policy and health sector plan, Child injury prevention committee formed at Prime Minister's office and Child drowning has been included in regular government child health programme. CIPRB has also added a value in the global efforts in child injury prevention.

Conclusions A champion organisation can make a significant change in putting a neglected health issue into a priority agenda competing with other health problem. So it is important to identify a champion organisation or form a platform with champion people to address child injury problem in low and middle-income countries.

\section{ONTOLOGY APPROACH FOR ANALYSIS OF SAFETY INCIDENTS IN URBAN TRANSPORT}

Alok Nikhil Jha, Niladri Chatterjee, Geetam Tiwari. Indian Institute of Technology Delhi, India

\subsection{6/injuryprev-2016-042156.711}

Background The safety measures in Transportation systems are increasing in Roads, Rails design and operations, Vehicles' ergonomics and new safety measures. United Nations General Assembly proclaimed a Decade of Action for Road Safety 2011-20 with a goal of stabilising and reducing road traffic fatalities around the world. Safety acquires a relevance when accounting for transportation systems especially to Public Transport

Problem Automotive industry adds latest safety elements inside vehicles, But the accidents and injury still exists. With yearwise planning for Safety Management we still lack a knowledge base of semantic relationship of various aspects of Safety in Urban Transport.

Results (Effects and Changes) The aim is to organise available data and create Ontology for safety of Urban Transport Safety. It will be developed as structure by analysis and formulate the knowledge that will be validated once on domain constraints and attributes. Ontologiy allow the creation of annotations in which the information is organised as a computer readable and understandable content.

Study at various levels in unsupervised, semi-supervised and supervised learning approaches are done to identify the best fit. The ontology will provide framework for hanging rules, probabilistic information and knowledge schema to search, add, update and extract or disseminate information, that can be applied in prediction, behavioural aspects, other issues in a common format globally along with decision making at real time in case of fatalities.

Conclusions The Ontology for Safety Management in Public Transport will assist Policy makers, authorities, researchers for creating a baseline of semantics of safety in a Transport System.This standardised system can be used in data sharing, analysis and knowledge management.Semantic relationship among various parameters and dependency in multiple domain will make this approach scalable and acceptable globally.

Prob The major concerns came up was regarding management of these information and utilisation of these information thus collected. The utilisation process should be strategically aligned so that multiple outputs can be created and used in various analysis of Safety

Governments are increasingly establishing restrictive regulations to improve safety on roads and public transportation systems in Urban and Rural context, with an aim of making travel safer.

Ontologies have been proposed to solve the problems that arise from using different terminology to refer to the same concept or using the same term to refer to different concepts.

The identified approach aims to develop a safe system semantically interrelated that can account all parameters of Safety in public transport.

\section{A DECADE OF INJURY AND VIOLENCE PREVENTION IN REPUBLIC OF MACEDONIA}

Fimka Tozija. Institute of Public Health of Republic of Macedonia, Skopje, Republic of Macedonia

10.1136/injuryprev-2016-042156.712

Background Violence and injuries are serious public health problem in Republic of Macedonia. Department for violence and injury control and prevention has been established in the Institute of Public Health of Republic of Macedonia in 2004 as a lead agency in health sector. The main objective is to present the achieved activities in addressing this problem in a period of one decade.

Methods Public health approach has been applied to assess the implementation of effective national policy interventions for violence and injury prevention and WHO Resolution EUR/RC55/R9 implementation.

Results Country responded to a European Council Recommendation of 31 May 2007. Violence and injury were set as priority in the Biannual collaborative agreements signed between the Ministry of Health and World Health Organisation. Specific national policies for road safety and domestic violence prevention have been implemented and amended legislation related with family violence and road safety Inter-ministerial National Coordination Body for family violence prevention and National coordination body for road safety were established. Report on violence and health in Macedonia and guide for prevention and Evaluation Report of the emergency medical services in Macedonia were prepared, Strategy for family violence, Law for prevention and protection of family violence, Protocol for family violence prevention and Strategy for Road Safety were adopted. WHO TEACH VIP capacity building was applied. Resolution EUR/ RC55/R9 was successfully implemented in Macedonia with $82 \%$ of 99 effective interventions, compared to European Region median score of $73 \%$ and much lower mortality of injuries with SDR 28/100000.

Conclusions Significant achievments in this decade are in national policy development, injury surveillance, capacity-building, multisectoral collaboration and evidence-based emergency care. Future challenges are evidence-based interventions for safety promotion and reducing socioeconomic inequalities. 